Research Article

\title{
Autonomic Function Correlates with Microflora Compositions in a Small Cohort of Patients with Autism Spectrum Disorder: Preliminary Clinical Data and Discussion
}

Daniel Goyal ${ }^{1,2,{ }^{*}}$, Fatma Mansab, James Neil ${ }^{3}$, Jaleel Miyan ${ }^{2}$

1. Department of Medicine, St Bernard's Hospital, Gibraltar; E-Mail: daniel.goyal@gha.gi

2. Faculty of Biology, Medicine \& Health, Division of Neurosciences \& Experimental Psychology, The University of Manchester, United Kingdom; E-Mail: j.miyan@manchester.ac.uk

3. Centre for Nutrition Education and Lifestyle Management (CNELM), London, United Kingdom; E-Mail: james@cnelm.co.uk

* Correspondence: Daniel Goyal; E-Mail: daniel.goyal@gha.gi

Academic Editors: Jenny Berrío and Bart Ellenbroek

Special Issue: $\underline{\text { On the Role of Early-life Neuroinflammation in Neuropsychiatric Disorders }}$

OBM Neurobiology

2019 , volume 3 , issue 2

doi:10.21926/obm.neurobiol.1902031
Received: March 05, 2019

Accepted: June 26, 2019

Published: June 28, 2019

\begin{abstract}
Background: Research into the effects of microflora on health and in disease has increased recently. Such enthusiasm seems well placed. Should the preliminary studies translate through the larger, more powerful studies, the medical community may well have an entirely new platform from which to intervene for health and against disease.

Methods: Delineating the pathways involved in the microflora and host interaction is likely to yield further avenues for intervention. Here we examine autonomic function and correlate this to compositional stool analysis (via qPCR) in 11 children with autism.

Results: Results show that there is a significant correlation between vagal tone and the proportion of firmicutes in the stools of patients with autism (Spearman's Ro $=0.782, p=$ $0.004, n=11)$.
\end{abstract}

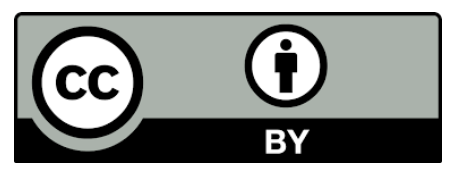

(C) 2019 by the author. This is an open access article distributed under the conditions of the Creative Commons by Attribution License, which permits unrestricted use, distribution, and reproduction in any medium or format, provided the original work is correctly cited. 
Conclusions: These preliminary results suggest there is a direct effect of microflora on neurological function in patients with autism. More powerful studies are required to advance this promising field further.

\section{Keywords}

Autism; autism spectrum disorder; microflora; autonomic function; vagal tone; neurological; gut-microbiome-brain; neuroinflammation; neuropsychiatry; firmicutes; clostridia

\section{Introduction}

Our understanding of the human microflora, its development and its impact on health remains in its infancy. It seems from the evidence available that from birth through to the early years the human microflora is still under development $[1,2]$. It also seems, from the evidence available, that there are a host of environmental factors that can affect microbiome composition [3]. There remains a lack of powered population studies in this area.

Microflora has been linked to a number of systemic diseases and host health [4-6]. Again, the research is currently quite limited. The prospect that we are then forced to consider, is that should the composition of the individual microflora correspond to disease activities, and the infant gut microflora is in extensive flux, then there is the real possibility of disease prevention and health promotion through insuring microflora compositions are conducive to health in infancy. This has potential ramifications for birthing environments, the use of unintentional antimicrobial agents (e.g. household products, pesticides, etc.) and of course intentional antibiotic use. There is also the possibility of novel therapeutic targets as we understand the interconnectedness of the microflora and neuroimmune systems.

We are quite distant from such a realisation in relation to our current knowledge base. There are though a number of large population-based studies underway in relation to microflora and health, and a number of studies examining the intricacies of microflora and host interactions. Quite soon we may have a surge of powerful studies to accelerate this area. Likely there will be genetic, immunogenic and possibly direct neurogenic considerations in determining what is a healthy/unhealthy microflora and the same considerations in determining the host's selection process. One expects that such considerations will be successful if determined on an individual to individual basis. Again, technology will need to keep pace with knowledge.

Treatment of disease associated with microflora abnormalities is somewhat different. Once a disease has developed and a divergent (from the population mean) microflora composition or species has been identified, it is reasonable to attempt disease modification through manipulation of the patient's microflora back towards 'normality'. That is, it is supposed that investigators need not wait for a detailed understanding of what constitutes 'typical' microflora development or the clarification provided by population studies pertaining to microflora changes relating to the onset of disease, prior to investigating the potential positive response to a systemic disease through manipulation of microflora. It may be enough to simply identify the divergence of microflora compositions from population norms in systemic disease(s) and to then attempt 'normalisation' of microflora and measure the effects on systemic disease. It is at this point we entertain the 
possibility that an abnormal microflora composition is driving a neuroinflammatory response in patients with autism spectrum disorder (ASD), and that this is a potential point of intervention.

In autism both a neuroinflammatory response and abnormal microflora compositions have been identified in a number of human studies. The presence of a neuroinflammatory response is quite convincing. Both post-mortem and CSF studies have demonstrated a pro-inflammatory cytokine profile and microglial activation in humans with autism [7, 8]. Microflora abnormalities in patients with ASD have been identified in a number of trials, albeit more powerful studies are awaited [9].

The supposition seems sound: abnormal microflora compositions can worsen a neuroinflammatory response. At a basic level the human microflora serves to stimulate the host immune system [10]. The mechanisms by which the host regulates such immunogenic potential remain unknown. Regardless, it is conceivable that the acquisition of a particularly immunogenic microflora species in infancy/early childhood or perhaps a failure in the regulatory mechanisms of the host, lead to an excessive pro-inflammatory microflora composition in patients with autism, thus driving a neuroinflammatory response.

A direct causal relationship between microflora abnormalities and neuroinflammation in ASD has not yet been established. A large population-based longitudinal study seems unavoidable if we are to investigate this further. Nonetheless, identifying microflora deviations from population norms and studying the potential associated effects of such abnormalities on the ASD host biology is useful. Perhaps there may be a more objective measure of response to 'improving' microflora compositions in ASD patients, and perhaps we may identify novel therapeutic targets to affect improved outcomes in patients with ASD.

\section{Methods and Results}

Included in this discussion paper is preliminary data from a retrospective analysis of autonomic function profiles and quantitative polymerase chain reaction (qPCR) stool analysis in children with ASD.

Autonomic profiles were extracted from a database of children with a diagnosis of ASD. Out of 227 patients with ASD who attended the outpatient clinic, 11 had autonomic profiles undertaken within one month of qPCR stool analysis and were free from antibiotics for three months.

A direct comparison was made between Vagal Tone as identified on autonomic testing and proportion of the firmicutes phyla present in the qPCR stool analysis.

Certain species from both the bacteriodetes and firmicutes phyla were also measured as part of the qPCR stool analysis. In previous work we have identified a potential shift towards the firmicutes phyla in patients with ASD (unpublished data). As the hypothesis here pertains to compositional alterations in microflora at the phyla level, no further analysis of individual species or group microflora were undertaken.

Autonomic profiling was undertaken using the Neuroscope method as previously described [11]. QPCR analysis was undertaken at Metametrix Laboratories through an in-house 16s DNA sequencing technique [12].

The results are represented in the scatter graph (Figure 1). There was a direct correlation between vagal tone and firmicutes levels (Pearson's correlation $=0.630, p=0.038, n=11$; Spearman's Ro $=0.782, \mathrm{p}=0.004, \mathrm{n}=11$ ). 


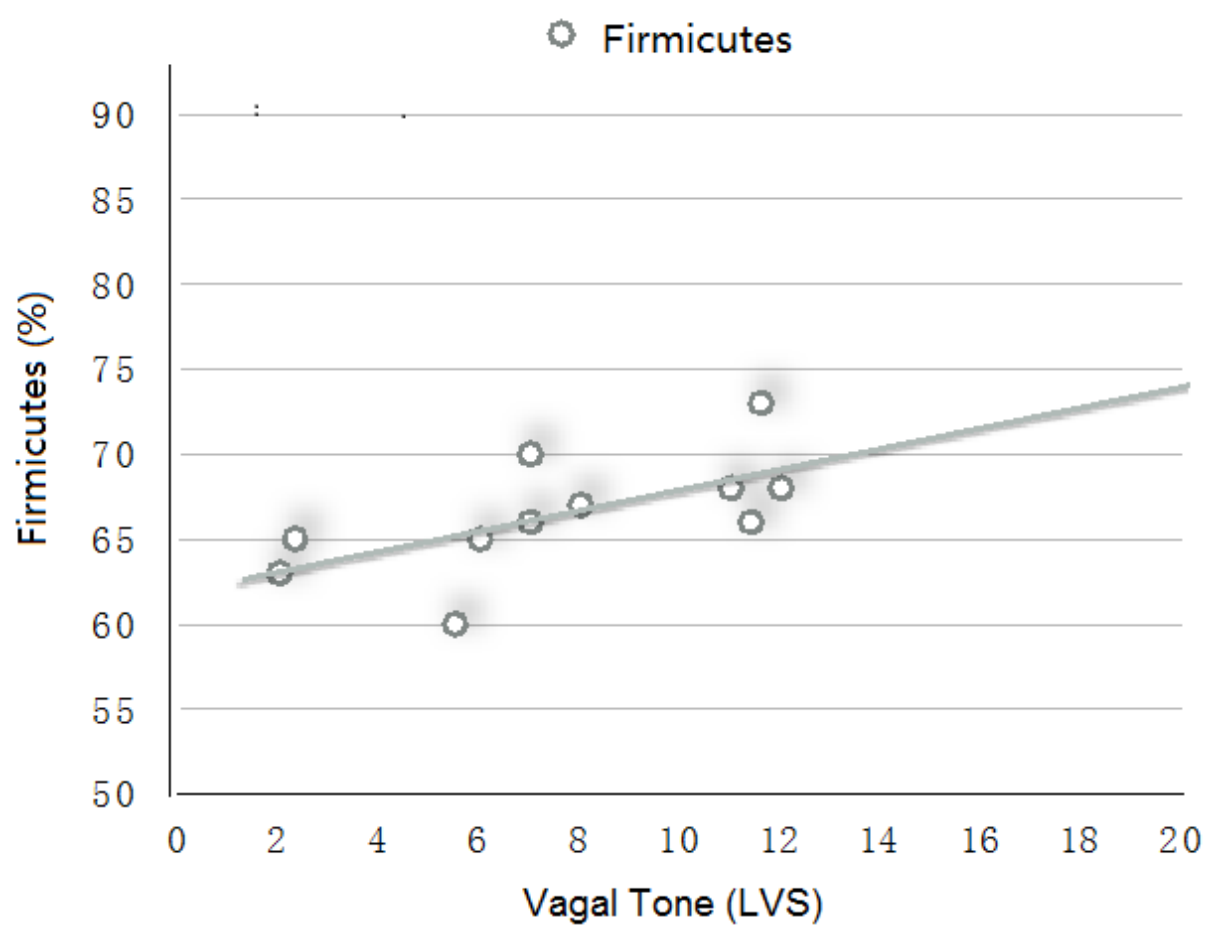

Figure 1. Scatter graph showing firmicutes proportion (\%) versus Vagal Tone (standardised units). Best fit line highlights the correlation between increasing firmuctes and increasing vagal tone.

\section{Discussion}

The association between autism and microflora has been explored by a number of investigators (eloquently summarised in the review paper Ding et al [13]). Most studies focus on species variation as opposed to compositional phyla alterations. As such, it is not possible to extrapolate whether or not the increased firmicutes ratio identified here is a consistent finding. In saying that, the clostridia species- a member of the firmicutes phyla - has been the most commonly implicated species, and in particular the quite robust investigation undertaken by Williams et al 2011 also identifies higher levels of clostridia in their ASD patient group versus controls [14].

There has also been a small clinical trial reporting a positive behavioural effect in patients with autism following treatment targeted at the microflora abnormalities [15], which has been recently supported by a clinical trial examining the behavioural effects of faecal transplants in patients with autism [16]. The studies are inconsistent in regards to technique and also the level at which microflora are studied - phyla, genus, species or sub-species -, but nonetheless, an intriguing line of enquiry. Larger clinical trials are awaited.

The data presented here was a targeted analysis in patients with autism of the phyla microflora composition versus the key autonomic measure, vagal tone. It is an interesting dataset, raising further the possibility of the much hypothesised gut-microbiome-brain connection. The limited analysis suggests there is a relationship between human gut flora and autonomic function. That is, as the firmciutes level increases, the parasympathetic nervous system increases in activity.

There are a number of possible explanations for this positive correlation. Perhaps, the firmicutes phyla is detected (through cytokines, endotoxins or perhaps even antigenic detection at the gut wall) and the brain's response is to increase the cholinergic anti-inflammatory pathway 
(i.e. to increase vagal tone). Therefore, in this regard the vagal response is simply an antiinflammatory response to a pro-inflammatory microflora composition. Perhaps though, the microflora selection process occurs via efferent and afferent pathways in the autonomic nervous system, and the association between firmicutes and vagal tone is simply the detection of this monitoring and regulatory activity of the brain.

\section{Limitations and Future Studies}

The dataset is limited in number and there is possible selection bias. The effects may not be unique to autism, as there were no other conditions or controls tested. The results do though concur with current hypotheses in the area, that microflora and brain interact - are interdependent.

Certainly, a repeat analysis with greater numbers of patients would be worthwhile. It is suspected that whilst the relationship between microflora and autonomic nervous system activity may well be a general finding - not specific to autism -, it is likely to be more useful to investigate autonomic function and microflora relationships in conditions where microflora abnormalities are likely to be important in disease activity.

\section{Conclusion}

Here, we present some preliminary clinical data in regards to microflora and autism, highlighting a possible mechanism or connection between the microflora and nervous system.

The results of qPCR and autonomic profile testing were analysed in 11 patients with ASD. Despite the low study numbers and possible selection bias, both parametric and non-parametric analysis demonstrated a significant correlation between the firmicutes phyla in stool and parasympathetic activity. These results suggest a connection between microflora and brain.

Further studies are warranted to help determine how microflora is regulated by the neuroimmune system. Understanding the effects of microflora compositions, species groups and specific species on health and disease may well lead to a new platform for disease prevention and intervention.

\section{Author Contributions}

Daniel Goyal originated the hypothesis, designed the methodology, conducted the study and wrote the majority of the article.

Fatma Mansab developed the hypothesis further, specifically providing immunological expertise, as well as helping to draw the conclusions and write the article.

James Neil provided the statistical expertise and designed the programme to extract data from patient files.

Jaleel Miyan provided guidance and support during the development of the hypothesis, methods, completing the study and generating the publication.

\section{Competing Interests}

The authors have declared that no competing interests exist. 


\section{References}

1. Scholtens PA, Oozeer R, Martin R, Amor KB, Knol J. The early settlers: intestinal microbiology in early life. Annu Rev Food Sci. Technol. 2012; 3: 425-447.

2. Koenig JE, Spor A, Scalfone N, Fricker AD, Stombaugh J, Knight R, et al. Succession of microbial consortia in the developing infant gut microbiome. P Nat Acad Sci USA. 2010; 108: 4578-4585.

3. Collado MC, Cernada M, Baüerl C, Vento M, Pérez-Martínez G. Microbial ecology and hostmicrobiota interactions during early life stages. Gut Microbes. 2012; 3: 352-365.

4. Carlson AL, Xia K, Azcarate-Peril MA, Goldman BD, Ahn M, Styner MA, et al. Infant gut microbiome associated with cognitive development. Biol Psychiatry. 2018; 83: 148-159.

5. Wang BH, Yao MF, LV LX, Ling ZX, Li L. The human microbiota in health and disease. Engineering. 2017; 3: 71-82.

6. Tanaka A, Nakayama J. Development of the gut microbiota in infancy and its impact on health in later life, Allergol Int, 2017; 60: 515-522.

7. Goyal DK, Miyan JA. Neuro-immune abnormalities in autism and their relationship with the environment: a variable insult model for autism. Front Endocrinol (Lausanne). 2014; 5: 29.

8. Pardo CA, Vargas DL, Zimmerman AW. Immunity, neuroglia and neuroinflammation in autism. Int Rev Psychiatry .2005; 17: 485-495.

9. Li Q, Han Y, Dy ABC, Hagerman RJ. The gut microbiota and autism spectrum disorders. Front Cell Neurosci. 2017; 11: 120.

10. Postler TS, Ghosh S. Understanding the holobiont: how microbial metabolites affect human health and shape the immune system. Cell Metab. 2017; 26: 110-130.

11. Ming X, Julu POO, Brimacombe M, Connor S, Daniels ML. Reduced cardiac parasympathetic activity in children with autism. Brain Dev. 2005; 27: 509-516.

12. Radjabzadeh, D., Uitterlinden, A.G. and Kraaij, R. Microbiome measurement: Possibilities and pitfalls. Best Pract Res Clin Gastroenterol. 2017; 31: 619-623.

13. Ding HT, Taur Y, Walkup JT. Gut microbiota and autism: key concepts and findings. J Autism Dev Disord. 2017; 47: 480-489.

14. Williams BL, Hornig M, Parekh T, Lipkin WI. Application of novel PCR-based methods for detection, quantitation, and phylogenetic characterization of Sutterella species in intestinal biopsy samples from children with autism and gastrointestinal disturbances. Mbio. 2012; 3: e00261.

15. Sandler RH, Finegold SM, Bolte ER, Buchanan CP, Maxwell AP, Väisänen ML, et al. Short-term benefit from oral vancomycin treatment of regressive-onset autism. J Child Neurol. 2000; 15: 429-435.

16. Kang DW, Adams JB, Coleman DM, Pollard EL, Maldonado J, McDonough-Means S, et al. Longterm benefit of microbiota transfer therapy on autism symptoms and gut microbiota. Sci Rep. 2019; 9: 5821. 


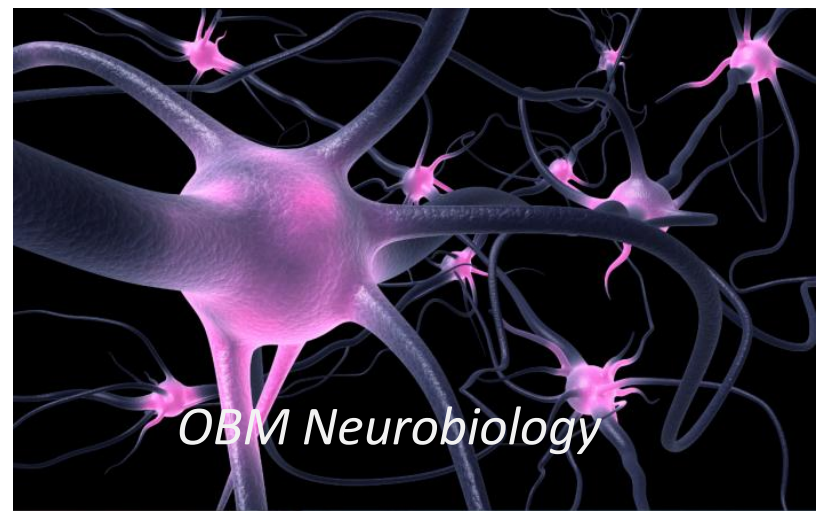

Enjoy OBM Neurobiology by:

1. Submitting a manuscript

2. Joining volunteer reviewer bank

3. Joining Editorial Board

4. Guest editing a special issue

For more details, please visit:

http://www.lidsen.com/journals/neurobiology 